\title{
Spermatogenesis following syngeneic testicular transplantation in Balb/c mice
}

\author{
Pengpeng Ma, Yehua Ge, Shali Wang, Jing Ma, Shepu Xue and Daishu Han \\ Department of Cell Biology, Institute of Basic Medical Sciences, Chinese Academy of Medical Sciences and Peking \\ Union Medical College, Beijing 100005, China
}

Correspondence should be addressed to Daishu Han; Email: daishu@public.bta.net.cn

\begin{abstract}
Transplantation of spermatogonial stem cells in cross-species has been widely used to study the function of Sertoli cells and the effect of phylogenetic distance between donor and recipient animals on the outcome of spermatogonial transplantation, whereas there have been only a few reports on the transplantation of testis tissue. The objective of the present study was to examine the development of grafted testes and the kinetics of spermatogenesis following syngeneic testicular transplantation in both male and female recipient Balb/c mice in an effort to establish an in vivo culture system and to compare the effects of host sex on spermatogenesis. The testes from 5-day-old Balb/c mice were transplanted under the dorsal skin of four-week-old mice. Twenty male and twenty female Balb/c mice were used as the hosts and each host received 4 grafts. The recipient mice were killed at 1, 2, 3, 5, 7, 9, 12 and 15 weeks after transplantation. The graft survival rate and graft size were measured. The status of spermatogenesis was assessed by histological analyses. The expression of the spermatid-specific Protamine-2 gene was examined by RT-PCR. Overall, $70.3 \%$ of the testicular grafts in male hosts and $67.2 \%$ in female hosts survived. All recovered grafts had increased in volume, some of them had increased by more than 30 -fold. The architecture of the seminiferous tubules in female hosts appeared to be better than that in male hosts. The round spermatids were the most advanced germ cells until 15 weeks after transplantation, and no complete spermatozoon was observed in any of the grafts. The expression of protamine-2 was detected in grafts from 5 weeks posttransplantation in both male and female hosts, confirming that the spermatogenic cells differentiated into spermatids. In contrast to grafts, the testes of male hosts had a normal histological appearance. The results showed the schedule of spermatogenesis following syngeneic testicular transplantation in both male and female hosts. This model could be useful for further studies involving the endocrinology of the testis and the mechanisms of spermatogenesis.

Reproduction (2004) 128 163-170
\end{abstract}

\section{Introduction}

Spermatogenesis is a productive and highly organized cell differentiation process resulting in the production of unlimited numbers of sperm during adulthood. Mammalian spermatogenesis can be divided into three phases: spermatogonia renewal and proliferation, meiosis, and spermiogenesis (Clermont 1972). Throughout spermatogenesis, continuous proliferation and differentiation of the germ cells depend strictly on the microenvironment of the seminiferous tubule-interactions between germ cells and somatic cells are essential. In particular, Sertoli cells maintain continuous contact with germ cells, from spermatogonia to spermatozoa. Spermiogenesis appears to be particularly dependent on the interaction between germ cells and Sertoli cells (Russell 1993).

Many aspects of the mechanisms of spermatogenesis have remained elusive because of a lack of suitable in vitro or in vivo models. Numerous trials have been aimed at mammalian spermatogenesis in vitro by many researchers over decades. But spermatogonial stem cells cannot survive and differentiate out of the microenvironment formed by other testicular cells, especially Sertoli cells. Further refinement of the culture conditions is needed, and hopefully this will lead to in vitro expansion of spermatogenic stem cells.

In 1994, the technique of spermatogonial transplantation was reported, through which germ cells of a fertile mouse can be transplanted into the seminiferous tubules of an infertile mouse to develop donor cell-derived spermatogenesis (Brinster \& Zimmermann 1994). In the most successful transplantation, the recipient mouse could transmit the haplotype of the donor cells to progeny (Brinster \& Avarbock 1994). A significant and surprising extension of these studies was xenogeneic spermatogonial 
transplantation. Testis cells from transgenic rats were transplanted to the testes of immunodeficient mice, and complete rat spermatogenesis occurred in the recipient mouse, resulting in the presence of normal appearing rat spermatozoa in the epididymides of the recipient mice (Clouthier et al. 1996). However, defects in spermiogenesis of transplanted hamster testis cells occur in the recipient mouse, which may reflect a limited ability of endogenous mouse Sertoli cells to support fully the larger and evolutionarily distant hamster germ cell (Ogawa et al. 1999). The transplantation of germ cells from phylogenetically more distant species, including rabbits, dogs, pigs, bulls, horses and primates, into mouse testes does not result in spermatogenesis beyond the stage of spermatogonial proliferation (Dobrinski et al. 1999, 2000, Nagano et al. 2001), probably owing to the incompatibility of microenvironments.

The transplantation of testis within and between individual animals is another experimental endeavour that has a long history in reproductive biology (Goldstein et al. 1983, Johnson et al. 1996a,b, Nagler et al. 2001). In comparison with germ cell culture and spermatogonial transplantation, this approach can maintain microenvironment integrity and provide the accessibility that is essential for studying the function of testes and spermatogenesis. The host can be regarded as a living incubator and a culture system of testicular tissue. In combination with the experimental manipulation of the donor testis in vitro before transplantation to the recipient, testis grafting would be a useful model for studying the molecular mechanisms of spermatogenesis. A model with syngeneic testicular grafts in Fischer rats was established in 1996 (Johnson et al. $1996 a, b)$. In this model, syngeneic prenatal/neonatal testicular grafts were transplanted under the skin of the outer ear in adult inbred Fischer rats that had been castrated and hypophysectomized. Complete spermatogenesis was found in grafts transplanted into the tips of the hosts' outer ears. It has been reported that different mammalian testicular grafts that were transplanted under the dorsal skin of 6-week-old male immunodeficient $\mathrm{NCr}$ mice could produce mature sperm (Honaramooz et al. 2002). Recently, progeny from sperm obtained after ectopic grafting of neonatal mouse testes were produced (Schlatt et al. 2003).

Most testicular transplantation models that have been established were used to study the effects of hormones on the growth and functions of the transplanted testis.
In order to analyse the schedule of spermatogenesis after syngeneic testicular transplantation in an effort to establish an in vivo culture system within the same strain for studying the mechanisms of mammalian spermatogenesis, in the present study we have developed a model of grafting testis tissues within the same strain of Balb/c mice to investigate the spermatogenesis in transplanted testes. Meanwhile, in order to look at the effects of host sex on the spermatogenesis of grafts, we used both intact male and female mice as the hosts.

\section{Materials and Methods}

\section{Animals and donor testis preparation}

Balb/c mice used in the study were obtained from the Laboratory Animal Centre of Beijing University (Beijing, China). Animals were kept and bred in the colony room with water and chow available and were maintained under controlled conditions ( $12 \mathrm{~h}$ light: $12 \mathrm{~h}$ darkness, 50\% humidity, $22^{\circ} \mathrm{C}$ ). The day that litters were born was considered to be day 1 of life. Five-day-postnatal male Balb/c mice were used as the source of donor testes. Before being grafted into recipient mice, all testes were maintained in DMEM (Gibco, Grand Island, NY, USA) on ice for $10 \mathrm{~min}$. Comparable tissue pieces were fixed at the time of grafting as reference for graft development. All the measures taken for the mice were in accordance with approved guidelines (Guidelines for the Care and Use of Laboratory Animals) established by the Chinese Council on Animal Care.

\section{Testicular transplantation experimental surgery}

Four-week-old Balb/c mice $(\sim 10-12 \mathrm{~g})$ were used as hosts. They were divided into two groups (20 mice each group) according to sex. All of them were intact. They were anaesthetized by intraperitoneal injections of sodium pentobarbital ( $4 \mathrm{mg} / 100 \mathrm{~g}$ body weight), which proves sufficient to anaesthetize them for over $1 \mathrm{~h}$. For a minimally invasive technique, we have developed a syringe needle plunger system for grafting. With this method, a great number of transplantations can be carried out in a short space of time. The procedure for transplantation is shown in Fig. 1. The testis removed from the 5-day-old donor was placed into the tubing of a 16-gauge needle (ID $1.0 \mathrm{~mm}, \mathrm{OD} 1.2 \mathrm{~mm})$, then the needle penetrated the
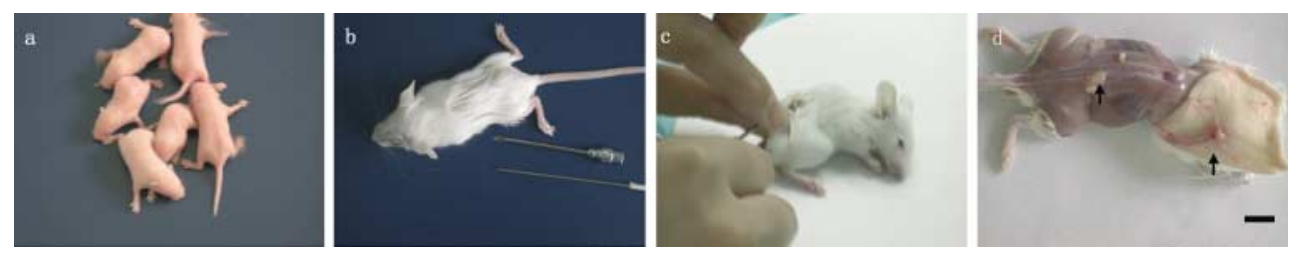

Figure 1 Transplantation of testis tissue. (a) Five-day-old donor Balb/c mice. (b) Four-week-old host, 16-gauge needle (ID $1.0 \mathrm{~mm}$, OD $1.2 \mathrm{~mm}$ ) and steel wire used in transplantation. (c) The donor testis is being injected under the dorsal skin, the wound in the host is self-sealing, and no sutures are necessary. (d) The size of the graft (arrows) was increased 3 weeks after transplantation. Bar $=1 \mathrm{~cm}$. 
dorsal skin of the host, and the testis was grafted in the site of destination under the skin by pushing a fine steel wire inside the tubing of the needle. Each host received 4 testicular grafts. The wound in the host is self-sealing, and no sutures are necessary.

\section{Morphometric and histological graft assessment}

Four host mice ( 2 males and 2 females) were killed at each time point at 1, 2, 3, 5, 7, 9, 12 and 15 weeks after transplantation. The status of graft survival was assessed with the method first described by Statter et al. (1988). In brief, the graft size was calculated by multiplying the length $\times$ width of the graft, both at the time of implantation (pre-size), and at the time the animal was killed (post-size). The graft size ratio (GSR) was equal to the post-size divided by the pre-size. Therefore, a GSR $>1$ indicates that the graft increased in size, GSR $=1$ indicates that there were no changes in graft size, GSR $<1$ indicates that the graft decreased in size and GSR $=0$ indicates that only scar tissue was found (rejection). For analysis of spermatogenesis of grafts, surviving grafts were removed from the hosts, fixed in Bouin's solution, dehydrated in a graded series of ethanol and subsequently embedded in paraffin. They were sectioned serially at $5 \mu \mathrm{m}$ and stained with haematoxylin and eosin. Tissue sections were qualitatively analysed for the degree of spermatogenic activity and for the most advanced stage of germ cell development achieved at the various time points analysed. Representative tissue sections were photographed. A total of $80-100$ seminiferous tubules were counted at each time point for calculating the percentage of seminiferous tubules containing different stages of spermatogenic cells and the percentage of seminiferous tubules with dilation of lumen. Meanwhile, the spermatogenesis of male host testes was also assessed by histological analysis.

\section{RNA isolation and reverse transcription (RT)-PCR}

For monitoring spermatogenesis of surviving grafts at the molecular level, the expression of Protamine-2 gene in transplanted testes was examined by RT-PCR at each time point after transplantation. Total RNA was isolated from surviving grafts with TRIzol reagent (Gibco) according to the manufacturer's instructions. The isolated RNA was treated with RNase-free DNase (Promega, Madison, WI, USA) at $37^{\circ} \mathrm{C}$ for $20 \mathrm{~min}$ to avoid contamination of genomic DNA, and was then reverse transcribed into cDNA in $50 \mu \mathrm{l}$ of reverse transcription reaction mixture containing $2 \mu \mathrm{g}$ RNA, $2 \mathrm{mM}$ random hexamers (Dingguo, Beijing, China), $2 \mathrm{mM}$ dNTPs, $0.01 \mathrm{M}$ dithiothreitol, $5 \mathrm{U}$ RNase inhibitor (Takara, Dalian, China), and $200 \mathrm{U}$ M-MLV reverse transcriptase (Gibco). The reaction was incubated at $37^{\circ} \mathrm{C}$ for $1 \mathrm{~h}$, and the enzyme was heat inactivated at $95^{\circ} \mathrm{C}$ for $5 \mathrm{~min}$. Two microlitres of cDNA synthesized were then amplified with the following primers for protamine-2
(GeneBank accession number: NM_008933): forward, 5'-ATG GTT CGC TAC CGA ATG A-3', reverse, 5'-TGA TGG TGC CTC CTA CAT TTC-3'. PCR cycle conditions were $94^{\circ} \mathrm{C}$ for $5 \mathrm{~min}, 94^{\circ} \mathrm{C}$ for $30 \mathrm{~s}, 58^{\circ} \mathrm{C}$ for $30 \mathrm{~s}, 72^{\circ} \mathrm{C}$ for $45 \mathrm{~s}$ for $30 \mathrm{cycles}$, and $72^{\circ} \mathrm{C}$ for $10 \mathrm{~min}$. PCR products were subjected to electrophoresis in $1.5 \%(\mathrm{w} / \mathrm{v})$ agarose gels, stained with ethidium bromide, and photographed.

\section{Statistical analysis}

Standard statistical methods were used for analysis of data. Probabilities of 0.05 or less were considered to be statistically significant. The data for the graft size ratio were summarized as means \pm S.D. In order to stabilize the group-to-group variability, a logarithmic transformation $\left(\log _{10}\right)$ was performed on the graft size ratios.

\section{Results}

\section{Graft survival rates and surviving graft sizes}

Transplantation of syngeneic 5-day-old mice testes under the dorsal skin of intact 4-week-old hosts resulted in growth and development. One week after testicular transplantation, eleven of the sixteen transplanted testes (69\%) were found under the dorsal skin of the host animals. Surviving rates of the grafts were $75 \%$ at 2 weeks posttransplantation, $69 \%$ at 3 weeks, $56.2 \%$ at 5 weeks, $75 \%$ at 7 weeks, $62.5 \%$ at 9 weeks, $69 \%$ at 12 weeks, and $75 \%$ at 15 weeks after transplantation. Overall, $70.3 \%$ of testicular grafts survived in male hosts, $67.2 \%$ survived in female hosts (Table 1 and Fig. 2). Most grafts had vascularized after transplantation. The grafts that did not vascularize might disappear later. There was no significant difference in graft survival rates between different sexed hosts. Most of the recovered grafts had increased in volume, some more than 30-fold (Table 1). Surviving graft sizes in male hosts increased significantly faster and were larger than in female hosts from 7 weeks post transplantation $(P<0.05)$.

\section{Evaluation of testicular differentiation by histological analysis}

In order to estimate the status of spermatogenesis of testicular grafts, the transplanted testes were removed and placed in Bouin's fluid. They were subsequently embedded in paraffin and stained with haematoxylin and eosin. Histological analyses showed the schedule of spermatogenesis in the testes after transplantation (Fig. $3 \mathrm{~A}-\mathrm{H}$ for male hosts, Fig. 3a-h for female hosts). The testes of 5-day-old mice contain abundant interstitial tissue and relatively thin seminiferous tubules which originally contain primitive type A spermatogonia and Sertoli cells (Fig. 3Y). No spermatogenic progression was observed in the 5-day-old mice testes. At one week after transplantation, the seminiferous tubules showed growth, and contained mainly spermatogonia and few preleptotene spermatocytes, indicating the initiation of spermatogenesis after grafting (Fig. $3 \mathrm{~A}$ and a). 
Table 1 Graft survival rates and graft sizes in different sex hosts after transplantation.

\begin{tabular}{|c|c|c|c|c|}
\hline $\begin{array}{l}\text { Time after } \\
\text { transplantation (weeks) }\end{array}$ & Recipient (sex) & Survival rate $(\%)$ & $\begin{array}{c}\text { GSR } \\
(\text { mean } \pm \text { standard deviation })\end{array}$ & $\begin{array}{c}\log _{\mathbf{1 0}} \mathbf{G S R} \\
(\text { mean } \pm \text { standard deviation })\end{array}$ \\
\hline \multirow[t]{2}{*}{1} & Male & 62.5 & $2.20 \pm 1.28$ & $0.26 \pm 0.25$ \\
\hline & Female & 75 & $2.06 \pm 1.26$ & $0.24 \pm 0.27$ \\
\hline \multirow[t]{2}{*}{2} & Male & 75 & $3.00 \pm 1.72$ & $0.40 \pm 0.21$ \\
\hline & Female & 75 & $3.22 \pm 1.47$ & $0.47 \pm 0.20$ \\
\hline \multirow[t]{2}{*}{3} & Male & 75 & $3.25 \pm 3.00$ & $0.49 \pm 0.28$ \\
\hline & Female & 62.5 & $3.23 \pm 1.13$ & $0.48 \pm 0.20$ \\
\hline \multirow[t]{2}{*}{5} & Male & 62.5 & $7.56 \pm 4.82$ & $0.78 \pm 0.41$ \\
\hline & Female & 50 & $7.34 \pm 4.23$ & $0.76 \pm 0.35$ \\
\hline \multirow[t]{2}{*}{7} & Male & 87.5 & $16.73 \pm 7.3$ & $1.22 \pm 0.43$ \\
\hline & Female & 62.5 & $9.77 \pm 3.92$ & $0.95 \pm 0.24$ \\
\hline \multirow[t]{2}{*}{9} & Male & 50 & $21.40 \pm 4.28$ & $1.32 \pm 0.10$ \\
\hline & Female & 75 & $14.00 \pm 4.25$ & $0.60 \pm 0.10$ \\
\hline \multirow[t]{2}{*}{12} & Male & 75 & $24.67 \pm 10.40$ & $1.36 \pm 0.18$ \\
\hline & Female & 62.5 & $8.42 \pm 2.54$ & $0.91 \pm 0.15$ \\
\hline \multirow[t]{2}{*}{15} & Male & 75 & $15.56 \pm 7.79$ & $1.12 \pm 0.30$ \\
\hline & Female & 75 & $5.28 \pm 4.08$ & $0.63 \pm 0.29$ \\
\hline
\end{tabular}

We observed the continual growth of the seminiferous tubules and the progression of spermatogenesis from 2 weeks to 5 weeks after transplantation. The presence of more spermatocytes was observed at 2 weeks and 3 weeks respectively, and round spermatids appeared at 5 weeks after transplantation. Two weeks after transplantation, $58.3 \%$ of the seminiferous tubules in male hosts and $58.6 \%$ in female hosts contained spermatocytes (Table 2). Round spermatids were seen in $6.2 \%$ of the seminiferous tubules in male hosts and in $7.9 \%$ in female hosts at 5 weeks after transplantation. At 12 weeks after transplantation, the number of seminiferous tubules that contained spermatocytes was $63.9 \%$ in male hosts and $90.3 \%$ in female hosts, but the number of seminiferous tubules that contained round spermatids was only $11.6 \%$ in male hosts and $18.3 \%$ in female hosts. Dilation of the seminiferous tubule lumen was found in both male and female hosts starting at week 2 after transplantation; the percentage of seminiferous tubules with dilation of the lumen was $18.3 \%$ in males and $6.9 \%$ in females. Starting at week 3 , dilation of the seminiferous tubule lumen became more prominent

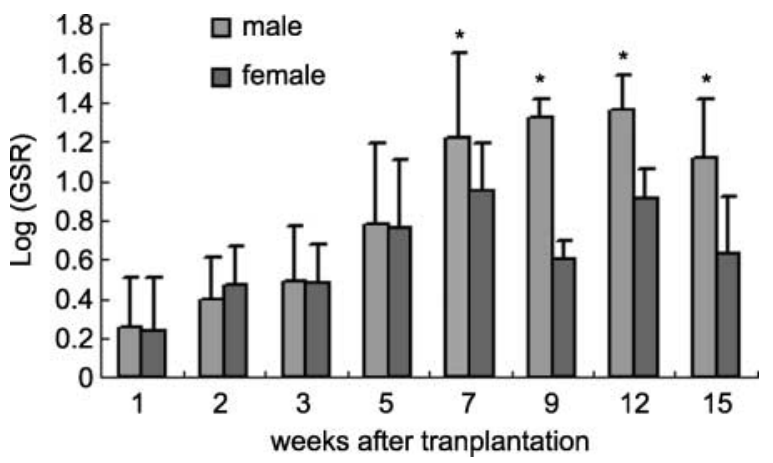

Figure 2 Changes in size of surviving grafts in different sex hosts after transplantation. The values are $\log _{10}$ transformed graft size ratios (postsize/presize). Each bar represents the mean \pm S.E.M. Asterisks indicate there is a significant difference in LogGSR of grafts between male and female hosts $(P<0.05)$. in male hosts, whereas much less dilation was observed in female hosts (Table 2). In contrast to male hosts, the architecture of most seminiferous tubules in female hosts appeared to be better. These results indicated that female mice might be more suitable to serve as hosts for testicular transplantation than male mice. However, no complete spermatogenesis was observed in any of the surviving grafts until 15 weeks after transplantation. In most surviving grafts, spermatocytes were the most advanced germ cells, round spermatids were observed only in some seminiferous tubules in both male and female hosts. In contrast to grafts, complete and normal spermatogenesis was observed in all male host testes after transplantation (Fig. 3I-VIII).

\section{RT-PCR}

Although the developmental stages of grafts were evaluated by histological analyses, it was essential to verify these results using other methods. We selected spermatidspecific Protamine-2 mRNA as a molecular marker of germ cell differentiation. We used RT-PCR to monitor the expression of protamine- 2 gene in testicular grafts. The RT-PCR results (Fig. 4) showed that protamine-2 was activated in grafts from 5 weeks posttransplantation in male and female hosts, suggesting that the spermatogenic cells in grafts could at least differentiate into round spermatids regardless of host sex. The fact that round spermatids could be found only in some grafts from 5 weeks posttransplantation whereas protamine-2 mRNA was detected in almost all grafts from 5 weeks to 15 weeks after transplantation is possibly caused by the section sites of the seminiferous tubules.

\section{Discussion}

Since the development of surgery, the possibility of testis transplantation has fascinated man for centuries (Attaran et al. 1966, Lee et al. 1971, Gittes et al. 1972, 

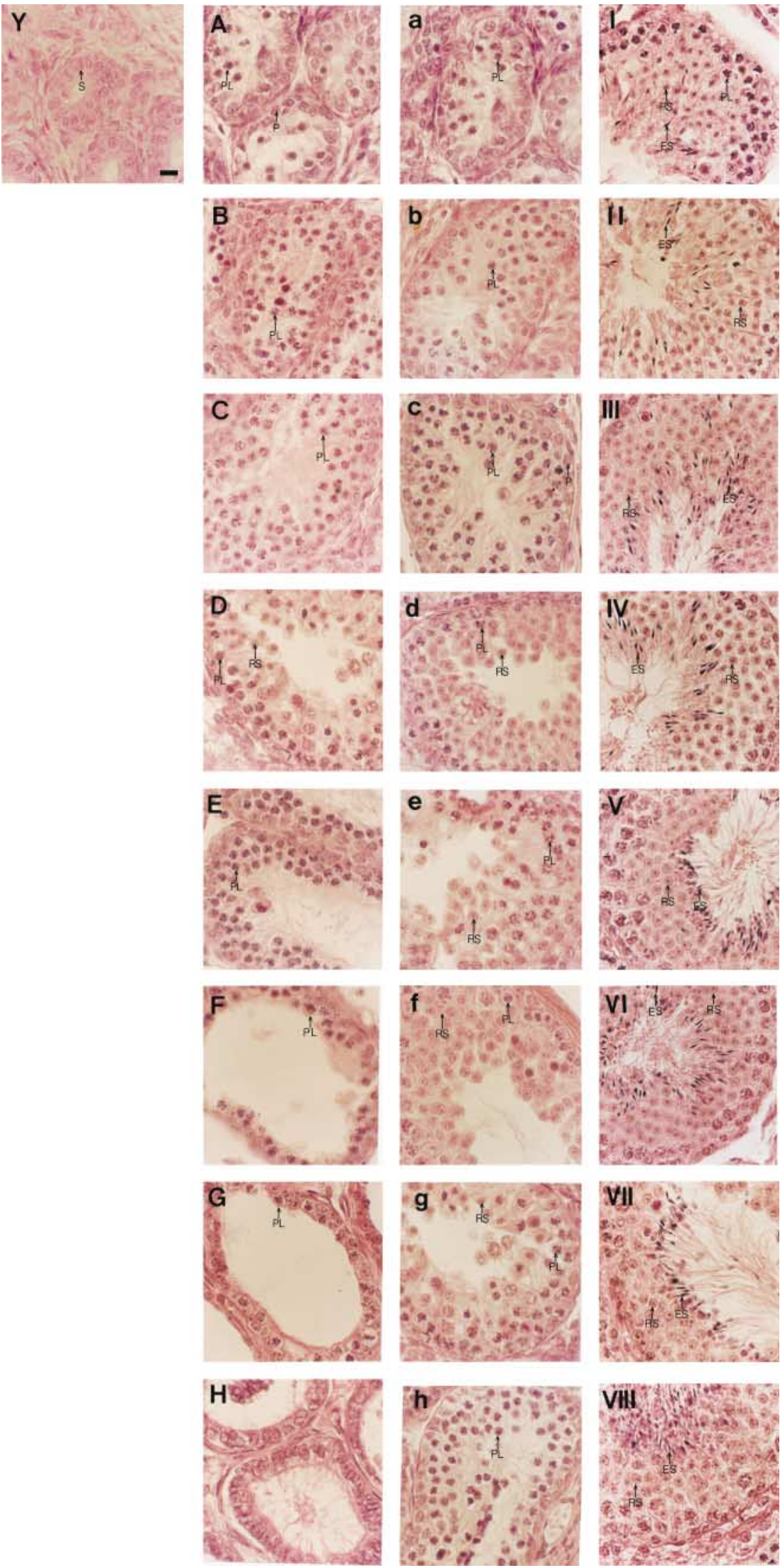
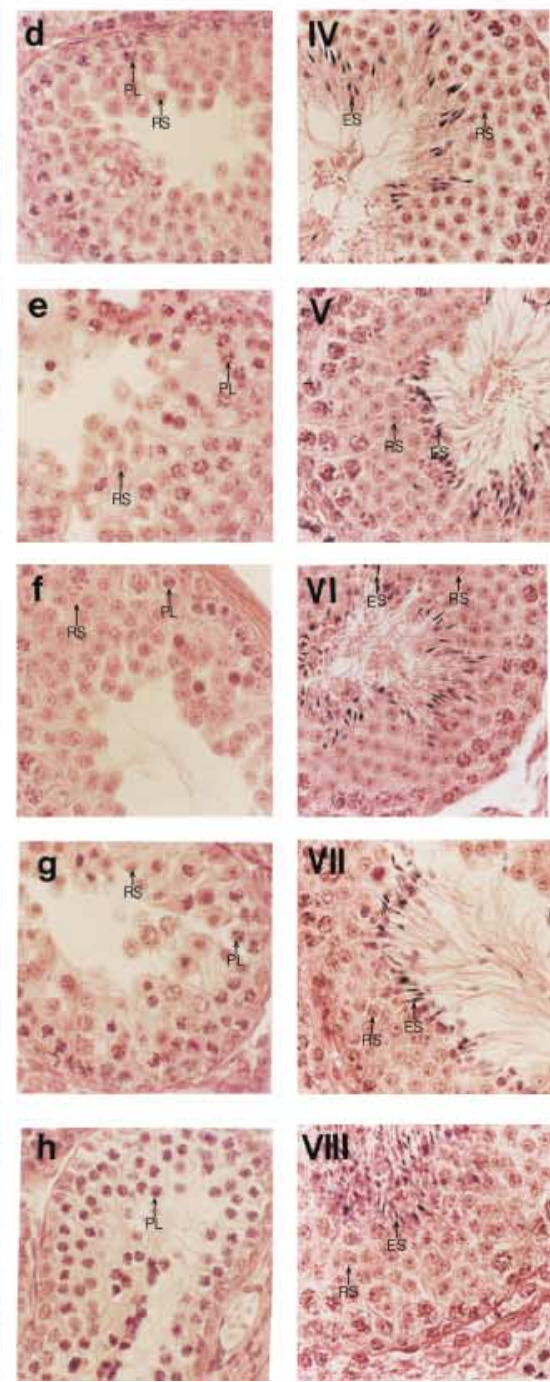
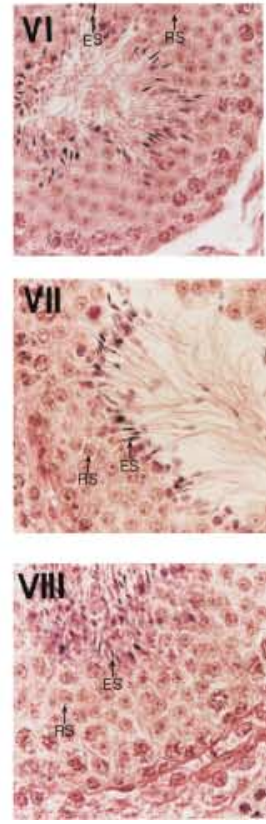

Figure 3 Histological examination of the donor testes and the host testes. (Y) Five-day-old postnatal mouse testis at the time of grafting. $(\mathrm{A}-\mathrm{H})$ Grafted testes in male hosts and $(\mathrm{a}-\mathrm{h})$ grafted testes in female hosts at 1, 2, 3, 5, 7, 9, 12 and 15 weeks after transplantation respectively. The histology shows the development of seminiferous tubules, epithelium and germ cells after transplantation. Spermatocytes are observed in almost all the seminiferous tubules of the grafts from 1 week after transplantation, and round spermatids are present at 5 weeks after transplantation in both male and female hosts. A distention of the tubular lumen is more prominent in seminiferous tubules of male hosts. (I-VIII) The male host testes at 1 , $2,3,5,7,9,12$ and 15 weeks after transplantation respectively. All stages of germ cells are present in the seminiferous tubules. The histological appearance of the host testis is similar to the mature testis, except for fewer elongated spermatids observed in the seminiferous tubule of the host testis at the first week after transplantation. Bar $=10 \mu \mathrm{m}$. S, Sertoli cell; P, spermatogonium; PL, primary spermatocyte; RS, round spermatid; ES, elongated spermatid. 
Table 2 Evaluation of the differentiation of transplanted testes in different sex hosts.

\begin{tabular}{lccccccc}
\hline \multirow{2}{*}{$\begin{array}{l}\text { Time after } \\
\text { transplantation } \\
\text { (weeks) }\end{array}$} & \multicolumn{3}{c}{ Male } & & \multicolumn{3}{c}{ Female } \\
\cline { 2 - 4 } \cline { 7 - 8 }$n$ & S\% & RS\% & D \% & & S\% & RS\% & D\% \\
\hline 2 & 58.30 & 0 & 18.30 & & 58.60 & 0 & 6.90 \\
3 & 50.00 & 0 & 74.00 & & 82.50 & 0 & 31.20 \\
5 & 55.00 & 6.20 & 75.00 & & 73.70 & 7.90 & 21.10 \\
7 & 73.20 & 7.40 & 78.70 & 80.60 & 9.70 & 33.90 \\
9 & 66.70 & 10.60 & 75.00 & & 86.60 & 11.30 & 17.10 \\
12 & 63.90 & 11.60 & 75.00 & & 90.30 & 18.30 & 16.10 \\
15 & 53.50 & 6.70 & 78.00 & 67.60 & 8.10 & 56.80 \\
\hline
\end{tabular}

$\mathrm{S} \%$, percentage of the seminiferous tubules containing spermatocytes; $\mathrm{RS} \%$, percentage of the seminiferous tubules containing round spermatids; $\mathrm{D} \%$, percentage of the seminiferous tubules containing a dilation of the lumen accompanied by a disorganized epithelium. A total of 80-100 seminiferous tubules were counted at each time point for calculating the percentage of the seminiferous tubules containing different stages of spermatogenic cells and the percentage of the seminiferous tubules with dilation of lumen.

Barten \& Newling 1996, Johnson et al. 1996a). Grafting of testis tissue was developed as a tool for androgen substitution in the 1950s (Deanesly 1954) and has subsequently been applied to study steroidogenesis and the functions of Sertoli or Leydig cells (Kuopio et al. 1989, Wilker \& Johnson 1995, Wilker et al. 1995, Johnson et al. 1996a,b), but few testicular transplantation models have been established for studying the mechanisms of spermatogenesis. The complete spermatogenesis in testes grafted into nude mice has been reported recently (Honaramooz et al. 2002, Schlatt et al. 2003), but there are few reports on spermatogenesis following syngeneic testicular transplantation in intact mice with normal immunity. In an effort to look at the effects of host sex on spermatogenesis of grafts, we chose intact male and female Balb/c mice as the hosts of testicular grafting in the present study. To our knowledge, this is the first report of syngeneic grafting in both intact male and female hosts. In our study, we have devised a minimally invasive method for grafting using a syringe needle plunger system. With this method, a great number of transplantations can be carried out in a short space of time.

The major hurdle in most instances of animal transplantation is tissue rejection by the recipient animal (Nagler et al. 2001). Statter et al. (1988) reported that testicular grafts from fetuses in the latter part of gestation as well as from several-days-postnatal animals grew and differentiated with minimal tissue rejection when they were implanted beneath the renal capsule of an adult male or female allogeneic host for 10 days without the benefit of immunosuppression. Previous studies (Johnson et al. $1996 a, b)$ have claimed greatest success with neonatal ( 0 day old) donor testes, when the number of spermatogenic stem cells is high. To extend the previous studies, we used 5-day-postnatal Balb/c mouse testes as the donor tissue, which contains only primitive A spermatogonia and Sertoli cells in the seminiferous tubules. After transplantation under the dorsal skin of 4-week-old syngeneic hosts, most grafts could grow and differentiate. Most of the surviving grafts were vascularized quickly after transplantation, as indicated by clearly visible arteries on the surface and interior of the organs (Fig. 1d). Turner (1938) transplanted whole rat testes into various sites in adult hosts, and proved that the intraocular and intramuscular sites provided the best vascular bed. An extensive vascular supply to the testicular transplants beneath the kidney capsule was also described (Kuopio et al. 1989). Our results indicated that a good vascular bed for grafted testis could be formed under the dorsal skin.

In the present study, most surviving grafts had grown in size, some of them increasing by 30 -fold. There was no significant difference in GSR between host sex by 5 weeks after transplantation, but surviving graft size in male hosts increased faster than in female hosts from 7 weeks posttransplantation. The difference in graft size is likely due to different hormone composition between male and female hosts. Further detailed investigation of the relationship between graft development and hormones in the hosts should expand the current understanding of the endocrinology of the testis.

Histological analysis showed that grafts under the dorsal skin were further developed in both male and female hosts. The diameters of the seminiferous tubules in the transplanted organs enlarged gradually, and the spermatogenic cells, which are mainly primitive type A spermatogonia and Sertoli cells in donor testes, were further developed after transplantation. Primary spermatocytes were observed
A
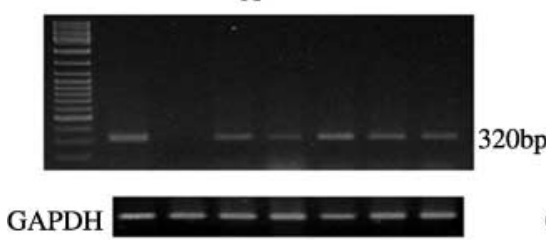

M

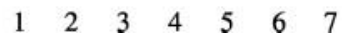

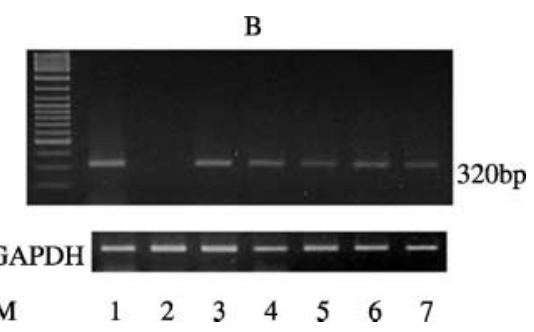

Figure 4 RT-PCR analysis of Protamine-2 mRNA in transplanted testes from (A) male and (B) female hosts. Results show protamine-2 was activated in grafts from 5 weeks after transplantation in both male and female hosts. The PCR products were subjected to electrophoresis in $1.5 \%$ agarose gels. Glyceraldehyde-3-phosphate dehydrogenase (GAPDH) serves as loading control. $\mathrm{M}$, marker; $1-7$, mRNA from testes of mature mice: 1, control; 2, 3 weeks post transplantation; 3, 5 weeks post transplantation; 4, 7 weeks post transplantation; 5, 9 weeks post transplantation; 6, 12 weeks post transplantation; 7, 15 weeks post transplantation. 
at week 1 after transplantation. This corresponds to the development schedule of testes in normal 2-week-old mice when the primary spermatocytes are the most advanced germ cell type, suggesting that grafts developed immediately after transplantation. Recently, Schlatt et al. (2003) transplanted neonatal mice testes under the dorsal skin of male immunodeficient $\mathrm{NCr}$ mice, and found that the first round of spermatogenesis had been completed by 4 weeks after transplantation, but at weeks 12 and 16, the number of seminiferous tubules containing spermatids was only about $40 \%$ and the number of fully matured germ cells remained low. In our study, 5-day-old mice transplanted testes could differentiate into round spermatids by week 5 after transplantation. In comparison with the results of Schlatt et al. (2003) the number of seminiferous tubules containing round spermatids was much lower $(11.6 \%$ in male hosts and $18.3 \%$ in female hosts 12 weeks after transplantation) and fully matured germ cells were not found in graft tissues in our study. These differences may be caused by two factors: (1) the temperature of the transplanted sites is lower in nude mice than in Balb/c mice and (2) neonatal donor testes may have greater developmental potential than 5-day donor testes after transplantation, because the spermatogenic stem cells rapidly decrease in volume during the period immediately after birth (Bellvé et al. 1977).

The dilation of the lumen was observed in seminiferous tubules of grafts in both male and female hosts starting at week 2 after transplantation, and many seminiferous tubules contained disorganized epithelium. In contrast to female hosts, dilation of the seminiferous tubule lumen was more prominent in male hosts. Interestingly, the architecture of the seminiferous epithelium of grafts in female hosts appeared better than in male hosts and there was less dilation of the lumen in the grafts in female hosts. Honaramooz and collegues have reported the dilation of the lumen in grafts. They postulated that the obvious absence of efferent ducts in the grafted tissue and the accumulation of fluid secreted into the seminiferous tubules account for the defects leading to disturbance of spermatogenesis (Honaramooz et al. 2002, Schlatt et al. 2003). The reason why grafts in female hosts had less dilation of the lumen in our study is unknown. It may be due to the fact that fluid flow secreted by Sertoli cells is decreased in female hosts but it is difficult to interpret. Nevertheless, female mice could be considered as more suitable hosts for testicular transplantation than male mice. In addition, the spermatogenesis of host testes was also assessed. Histological analysis indicated that testicular grafts had no significant effects on the spermatogenesis of the host testes.

In this study, we did not observe complete spermatogenesis until 15 weeks after transplantation in all grafts. Round spermatids were the most advanced germ cells observed in the seminiferous tubules of transplanted grafts. Turner (1938) transplanted whole testes from 1- to 21-dayold rats into various sites (anterior chamber of the eye, under the skin of the thoracic region and scrotum) in adult hosts. It was proved that testes transplanted into the anterior chamber of the eye and under the skin of the thoracic region resulted in high recovery of viable testicular tissues. However, mature sperm were only found in the testes transplanted to the scrotum. The only difference between the scrotum and the other sites is the temperature, suggesting that the temperature of the transplanted site could be the factor permitting later stages of spermatogenesis to appear in grafts (Chan et al. 1969). Several studies subsequently indicated that testicular grafts under the ear skin where the temperature is approximately the same as that of the scrotum did produce mature sperm (Johnson et al. 1996a,b). According to these reports, the failure of complete spermatogenesis in the present study should be due to the unfavourable temperature under the dorsal skin. Since round spermatids were observed in the grafts, the temperature probably has a greater effect on spermiogenesis. It should be noted that different newborn mammalian testicular grafts that were transplanted under the dorsal skin of 6-week-old male immunodeficient $\mathrm{NCr}$ mice could produce mature sperm (Honaramooz et al. 2002). The lack of hair in the nude mice hosts might result in a lower temperature under the dorsal skin, resulting in complete spermatogenesis in grafts. Considering the fact that spermatogenesis fails in cryptorchid testes, temperature might be a key factor during spermatogenesis, particularly in the transition from round spermatids to elongating spermatids.

In order to confirm the developmental stages of germ cells in grafts, we employed RT-PCR to examine expression of protamine-2 gene in transplants. The protamine-2 gene is expressed exclusively in postmeiotic, haploid spermatids (Oliva \& Dixon 1991), and can be used as a molecular marker to monitor spermatogenesis. In this study, protamine-2 mRNA was detected in some grafts from 5 weeks posttransplantation, confirming that the spermatogenic cells in the grafts did differentiate into round spermatids. It was reported that size changes of protamine-1 mRNA could be used as a molecular marker to monitor spermatogenesis in wild-type and mutant mice (Hecht et al. 1985). Our present study indicated that the protamine-2 transcript was also a useful molecular marker to monitor spermatogenesis in mouse. In addition, there was no significant difference in expression of protamine- 2 between different host sexes.

In summary, we have developed a reliable technique for the rapid transplantation of mouse testes, and with this method we transplanted the testes from 5-day-old Balb/c mice under the dorsal skin of syngeneic mice and analysed the development of the grafts. The results demonstrated that most grafts undergo further development, and that the spermatogenic cells can be differentiated to the round spermatid stage in some grafts. The development of the grafts in female hosts appeared to be better compared with male hosts, with less dilation of the lumen and less disorganized epithelium. Therefore, it is suggested that the 
female host may be more suitable for testicular transplantation. This is the first report of spermatogenesis in testes transplanted into Balb/c mice, and the first report of syngeneic grafting compared in both intact male and female hosts. This model could be useful in the study of the endocrinology of the testis and the mechanisms of spermatogenesis in combination with treatment of the donor testis in vitro before transplantation.

\section{Acknowledgements}

This work was supported by Special Funds for Major State Basic Reseach Project of China (grant no. G1999055901).

\section{References}

Attaran SE, Hodges CV, Crary LS Jr, Vangalder GC, Lawson RK \& Ellis LR 1966 Homotransplants of the testis. Journal of Urology 95 387-389.

Barten EJ \& Newling DWW 1996 Transplantation of the testis; from the past to the present. International Journal of Andrology 19 205-211.

Bellvé AR, Cavicchia JC, Millette CF, O'Brien DA, Bhatnagar YM \& Dym M 1977 Spermatogenic cells of the prepuberal mouse. Isolation and morphological characterization. Journal of Cell Biology 74 68-85.

Brinster RL \& Avarbock MR 1994 Germline transmission of donor haplotype following spermatogonial transplantation. PNAS 91 11303-11307.

Brinster RL \& Zimmermann JW 1994 Spermatogenesis following male germ cell transplantation. PNAS 91 11298-11302.

Chan F, Allison JE, Stanley AJ \& Gumbreck LG 1969 Reciprocal transplantation of testes between normal and pseudohermaphroditic male rats. Fertility and Sterility $20482-494$.

Clermont Y 1972 Kinetics of spermatogenesis in mammals: seminiferous epithelium cycle and spermatogonial renewal. Physiology Reviews 52 198-236.

Clouthier DE, Avarbock MR, Maika SD, Hammer RE \& Brinster RL 1996 Rat spermatogenesis in mouse testis. Nature 381 418-421.

Deanesly R 1954 Spermatogenesis and endocrine activity in grafts of frozen and thawed rat testis. Journal of Endocrinology $\mathbf{1 1}$ 201-206.

Dobrinski I, Avarbock MR \& Brinster RL 1999 Transplantation of germ cells from rabbits and dogs into mouse testes. Biology of Reproduction 61 1331-1339.

Dobrinski I, Avarbock MR \& Brinster RL 2000 Germ cell transplantation from large domestic animals into mouse testes. Molecular Reproduction and Development 57 270-279.

Gittes RF, Altwein JE, Yen SS \& Lee S 1972 Testicular transplantation in the rat: long-term gonadotropin and testosterone radioimmunoassays. Surgery 72 187-192.

Goldstein M, Phillips DM, Sundaram K, Young GP, Gunsalus GL, Thau R \& Bardin CW 1983 Microsurgical transplantation of testes in isogenic rats: method and function. Biology of Reproduction $\mathbf{2 8}$ 971-982.
Hecht NB, Bower PA, Kleene KC \& Distel RJ 1985 Size changes of protamine 1 mRNA provide a molecular marker to monitor spermatogenesis in wild-type and mutant mice. Differentiation 29 189-193.

Honaramooz A, Snedaker A, Boiani M, Scholer H, Dobrinski I \& Schlatt S 2002 Sperm from neonatal mammalian testes grafted in mice. Nature 418 778-781.

Johnson L, Suggs LC, Norton YM \& Zeh WC 1996a Effect of developmental age or time after transplantation on Sertoli cell number and testicular size in inbred Fischer rats. Biology of Reproduction 54 948-959.

Johnson L, Suggs LC, Norton YM, Welsh TH Jr \& Wilker CE $1996 \mathrm{~b}$ Effect of hypophysectomy, sex of host, and/or number of transplanted testes on Sertoli cell number and testicular size of syngeneic testicular grafts in Fischer rats. Biology of Reproduction $\mathbf{5 4}$ 960-969.

Kuopio T, Savouras PO, Pelliniemi LJ \& Huhtaniemi IT 1989 Transplantation of newborn rat testis under the kidney capsule of adult host as a model to study the structure and function of Leydig cells. Journal of Andrology 10 335-345.

Lee S, Tung KS \& Orloff MJ 1971 Testicular transplantation in the rat. Transplantation Proceedings 3 586-590.

Nagano M, McCarrey JR \& Brinster RL 2001 Primate spermatogonial stem cells colonize mouse testes. Biology of Reproduction 64 1409-1416.

Nagler JJ, Cloud JG, Wheeler PA \& Thorgaard GH 2001 Testis transplantation in male rainbow trout (Oncorhynchus mykiss). Biology of Reproduction 64 644-646.

Ogawa T, Dobrinski I, Avarbock MR \& Brinster RL 1999 Xenogeneic spermatogenesis following transplantation of hamster germ cells to mouse testes. Biology of Reproduction 60 515-521.

Oliva R \& Dixon GH 1991 Vertebrate protamine genes and the histone-to-protamine replacement reaction. Progress in Nucleic Acid Research and Molecular Biology 40 25-94.

Russell LD 1993 Morphological and functional evidence for Sertoligerm cell relationships. In The Sertoli Cell, pp 365-390. Eds LD Russell \& MD Griswold. Clearwater, FL: Cache River Press.

Schlatt S, Honaramooz A, Boiani M, Scholer HR \& Dobrinski I 2003 Progeny from sperm obtained after ectopic grafting of neonatal mouse testes. Biology of Reproduction 68 2331-2335.

Statter MB, Foglia RP, Parks DE \& Donahoe PK 1988 Fetal and postnatal testis shows immunoprivilege as donor tissue. Journal of Urology 139 204-210.

Turner CD 1938 Intraocular homotransplantations of prepubertal testis in rat. American Journal of Anatomy 63 101-159.

Wilker CE \& Johnson L 1995 Effects of donor age, host weight, and ear location on size of syngeneic testicular grafts. Journal of Andrology 16 (Suppl) 59.

Wilker CE, Ing NH, Welsh TH Jr \& Johnson L 1995 Syngeneic testicular grafts produce testosterone and express Sertoli cell genes characteristic of intact rats. Journal of Andrology 16 (Suppl) 56.

Received 31 December 2003

First decision 11 March 2004

Revised manuscript received 24 March 2004

Accepted 10 May 2004 